\title{
Dispersion properties of photonic crystal fibres
}

\author{
Bjarklev, Anders Overgaard; Broeng, Jes; Dridi, Kim; Libori, Stig E. Barkou
}

Published in:

Optical Communication, 1998. 24th European Conference on

Link to article, DOI:

10.1109/ECOC.1998.732471

Publication date:

1998

Document Version

Publisher's PDF, also known as Version of record

Link back to DTU Orbit

Citation (APA):

Bjarklev, A. O., Broeng, J., Dridi, K., \& Libori, S. E. B. (1998). Dispersion properties of photonic crystal fibres. In Optical Communication, 1998. 24th European Conference on (Vol. 1, pp. 135-136). IEEE. https://doi.org/10.1109/ECOC.1998.732471

\section{General rights}

Copyright and moral rights for the publications made accessible in the public portal are retained by the authors and/or other copyright owners and it is a condition of accessing publications that users recognise and abide by the legal requirements associated with these rights.

- Users may download and print one copy of any publication from the public portal for the purpose of private study or research.

- You may not further distribute the material or use it for any profit-making activity or commercial gain

- You may freely distribute the URL identifying the publication in the public portal 


\title{
DISPERSION PROPERTIES OF PHOTONIC CRYSTAL FIBRES
}

\author{
Anders Bjarklev (1), Jes Broeng (2), Kim Dridï (3), and Stig E. Barkou (4)
}

Department of Electromagnetic Systems, Technical University of Denmark, DTU Building 348, DK-2800, Lyngby, Denmark, Tel.: +45 4588 14 44, Fax: +45 4593 16 34, E-mail: (1) ab@emi.dtu.dk, (2) ib@emi.dtu.dk

Abstract: Approximate dispersion and bending properties of all-silica two-dimensional photonic crystal fibres are characterised by the combination of an effective-index model and classical analysis tools for optical fibres.

\begin{abstract}
Introduction
Together with the rapidly developing employment of wavelength division multiplexed optical communication systems, a renewed interest in the development of advanced optical fibres for new applications has been seen. The research primarily includes the further development of the mature silica fibre technology to handle amplification, dispersion compensation, nonlinearities etc. However, also completely new fibre concepts have been introduced over the past few years, among which we believe the photonic crystal fibre (PCF) [1] to be one of the most interesting.

The PCF is a silica fibre with a hexagonal array of air holes running down its length. It is particularly interesting from a waveguiding point of view, because it shows properties that are very different from standard optical fibres, including single-mode operation in a wavelength range from $337 \mathrm{~nm}$ to beyond $1550 \mathrm{~nm}$ [2]. In a recently published article [3], further experimental and numerical evidence for the unique single-mode properties of the PCF was presented, and it was stated that the dispersion properties of the fibre are expected to exhibit some unusual features. Since it is exactly these properties that may decide whether or not the PCF is potentially interesting for future communication system applications, we have in this letter taken up the challenge of predicting the dispersion properties of these new fibres.

The approach for this analysis is based on the combination of the approximate effective-index model [2] developed for PCFs and well-established propagation models for standard optical fibres.
\end{abstract}

\section{Modelling of the photonic crystal fibre}

The PCF waveguide is formed by a hexagonal array of air holes (with diameter d) embedded in a silica matrix, where the center-to-center spacing between the nearest air holes is generally refered to as the pitch, $\Lambda$.

For the calculation of the waveguiding properties of the fibre, point of reference is chosen in the effective-index model outlined in [2]. In this model, the waveguide consists of a core and a cladding region that have refractive indices $n_{c o s}$ and $n_{c l}$. The core is pure silica, but the definition of the refractive index of the microstructured cladding region is defined in terms of the propagation constant of the lowest order mode that could propagate in the infinite cladding material. This cladding mode field, $\psi$, is determined by solving the scalar-wave equation within a unit cell centered on one of the holes. By reflection symmetry the boundary condition at the cell edge (at radius $\Lambda / 2$ ) is $d \psi / d s=0$, where $s$ is the coordinate normal to the edge. The propagation constant of the resulting fundamental space-filling mode, $\beta_{\mathrm{FSM}}$, is used to define the effective index of the cladding as $\mathrm{n}_{\mathrm{efI}}=\beta_{\mathrm{FSM}} / \mathrm{k}$ (where $\mathrm{k}$ is the free-space propagation constant of light with wavelength $\lambda$ ). Now having determined the cladding- and core-index values, the approximate propagation properties of the PCF may be calculated as for a stepindex fibre with core index $n_{\mathrm{co}}$, core radius $\Lambda / 2$, and cladding index $n_{\mathrm{cl}}=n_{\mathrm{cffi}}$. The present cladding-mode model only deviate from the one described in [2], by the addition of a wavelength dependent refractive index for silica through the SelImeier formula [4]. Furthermore, the dispersion properties are calculated by numerical differentiation as described in [5].

In [2], the bending properties of the PCF was described by the introduction of a critical bend radius. However, in order to better characterise the unique PCF bending properties including both a low-wavelength and a long-wavelength bend-loss edge, we have here chosen to apply the bending loss formula described in [6].

\section{Comparison between standard fibres and PCFs}

To characterise the potential communication system applications of PCFs, it is natural to compare their properties to those of the standard optical fibre. Therefore, we first focus on the spectral spotsize variation of a PCF with a pitch of $2.3 \mu \mathrm{m}$, and an air-hole size given by $\mathrm{d} / \Lambda=0.40$ (fibre parameters comparable to those presented in [2]). The calculated PCF spotsize is shown in Figure 1 as a function of wavelength together with the spotsize of a standard stepindex fibre.

Fig. 1: Spotsize comparison between standard fibre and photonic crystal fibre

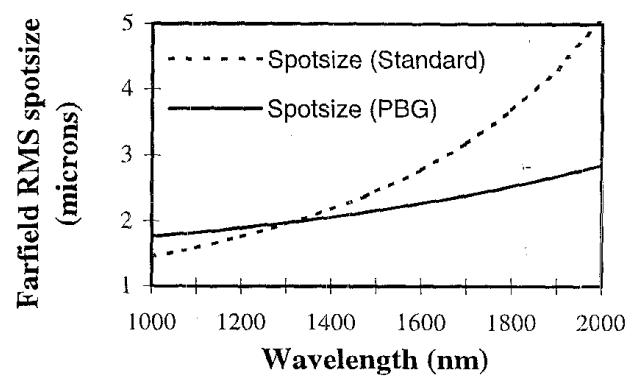

The standard fibre has the same core-cladding refractiveindex difference as the PCF at the wavelength $\lambda=1.3 \mu \mathrm{m}$, and a core diameter of $\Lambda$. An important observation from this comparison is that the PCF has a much weaker wavelength dependency than the standard fibre.

The transparency range of the PCF was in [2] described to be ultimately limited by bend-loss edges at short wavelengths and at long wavelengths. Applying the formula of 
[6], the bend loss is directly calculated from the Bessel function coefficients and propagation constant of the effective-index fibre. In the case, where the full length of the fibre is coiled at a radius of $6.0 \mathrm{~cm}$ (corresponding to dispersion-compensating fibre coils), the bending-loss values for different air hole dimensions are shown in Figure 2 for a PCF with $\Lambda=2.3 \mu \mathrm{m}$. Note that both the upper and the lower bend edge is found, which clearly is in contrast to the standard fibre (bend loss also shown in Figure 2 for the above mentioned step-index fibre).

Fig. 2: Bending loss properties for standard fibres and PCFs coiled with a $6.0 \mathrm{~cm}$ radius

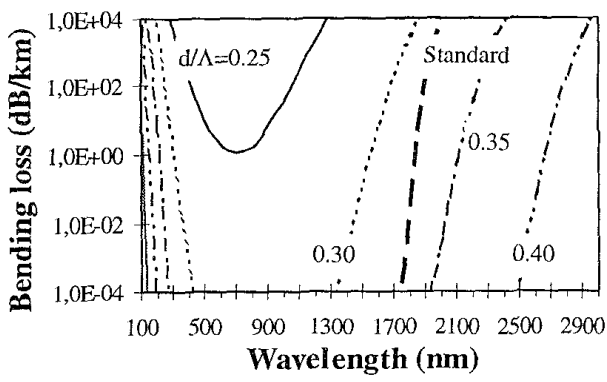

These results indicate that a large operational wavelength range (of interest to optical communications) is available with PCFs, which in addition have the perspective of providing very low scattering losses, because they may be fabricated from undoped silica.

\section{Dispersion properties of photonic crystal fibres}

After having indicated the unique properties of PCFs with regard to spotsize and loss, the most interesting question remains, namely how are the dispersion properties of these new fibres. To clarify this, point of reference is once more taken in the design outlined in [2-3], i.e., a PCF with a pitch of $2.3 \mu \mathrm{m}$. For such fibres with variable air hole filling fractions, the dispersion is shown in Figure 3 as a function of wavelength.

Fig. 3: Dispersion as a function of wavelength for PCFs with a fundamental cell diameter $\Lambda=2.3$ microns (curves for different ratios $d / \Lambda$ )

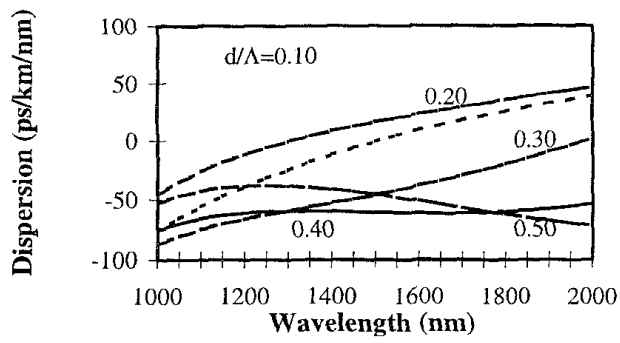

From Figure 3 we first note that for very small air filling fractions, e.g., when the influence of the air holes are strongly limited, the dispersion curve is expectedly very close to the material dispersion of pure silica (zero dispersion wavelength around $1.3 \mu \mathrm{m}$ ). As the diameter of the air holes is increased, the waveguide dispersion becomes increasingly strong, and we obtain a significantly reduced dispersion. It is here particularly interesting that an almost constant dispersion level around $-60 \mathrm{ps} / \mathrm{km} / \mathrm{nm}$ is predicted for a ratio $\mathrm{d} / \Lambda=0.40$.

These results indicate the interesting possibility of applying the PCFs as dispersion compensating or dispersion managed fibres for optical communication systems. Therefore, to investigate this option further, a number of different designs have been analysed. The most spectrally constant dispersion values was calculated for fibres with a relative air hole sizes around $d / \Lambda=0.40$. Some of the results are presented in Figure 4 , where very flat dispersion curves with values down below $-100 \mathrm{ps} / \mathrm{km} / \mathrm{nm}$ are seen.

Fig. 4: Dispersion as a function of wavelength for PCFs with a relative hole size of $d / \Lambda=0.40$ (curves for different pitch values: $1.4-3.2 \mu \mathrm{m}$ in steps of $0.3 \mu \mathrm{m}$ ).

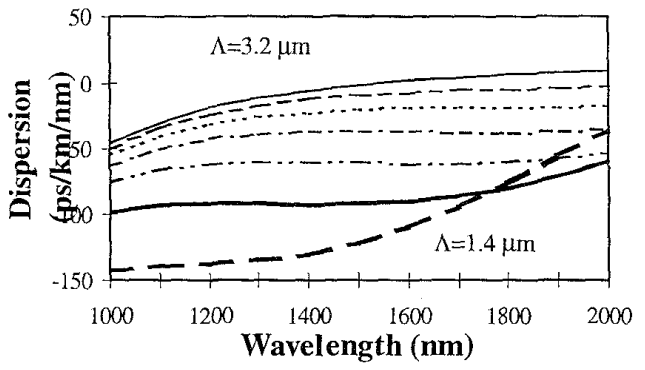

For the fibre parameter range used in Figure 4, it is noteworthy that the effective normalised frequency [2], $\mathrm{V}_{\mathrm{eff}}$, is in the range from 2.0 to 3.5 (smallest value for smallest pitch). This should be compared to the result of [3], where it is found that a PCF with $\mathrm{V}_{\text {eff }}<2.5$ could be expected to support only a single mode.

To the best of our knowledge, we believe for the first time to have predicted the dispersion properties of photonic crystal fibres. The results strongly indicate that these fibres have potential applications as dispersion managing components.

\section{References}

/1/ J.C.Knight, T.A.Birks, P.St.J.Russell, and D.M.Atkin, "All-silica single-mode optical fiber with photonic crystal cladding", Opt.Lett., Vol.21, 1996, pp.1547-49.

12/ T.A.Birks, J.C.Knight, and P.St.J.Russell, "Endlessly single-mode photonic crystal fiber", Opt.Lett., Vol.22, 1997, pp.961-963.

13/ J.C.Knight, T.A.Birks, P.St.J.Russell, and J.P.de Sandro, "Properties of photonic crystal fiber and the effective index model", J.Opt.Soc.Am., Vol.15, No.3, 1998, pp.748 752.

14/ J.W.Fleming, "Material dispersion in lightguide glasses" Electr.Lett., Vol.14, 1978, pp.326-328.

15/ A.Bjarklev, "Singlemode fibre characterisation and applications of mode coupling theory", Ph.D. thesis LD68, DTU, February 1988.

16/ J.Sakai, T.Kimura, "Bending loss of propagation modes in arbitrary-index profile optical fibersApplied Optics, Vol.17, 1978, pp.1499-1506. 